\title{
THE MINIMUM ALGEBRAIC CONNECTIVITY OF CATERPILLAR UNICYCLIC GRAPHS*
}

\author{
WAI CHEE SHIU ${ }^{\dagger}$, JI-MING GUO ${ }^{\ddagger}$, AND JIANXI LI ${ }^{\dagger}$
}

\begin{abstract}
A caterpillar unicyclic graph is a unicyclic graph in which the removal of all pendant vertices makes it a cycle. In this paper, the unique caterpillar unicyclic graph with minimum algebraic connectivity among all caterpillar unicyclic graphs is determined.
\end{abstract}

Key words. Algebraic connectivity, Caterpillar unicyclic graph, Characteristic polynomial.

AMS subject classifications. $05 \mathrm{C} 50$.

1. Introduction. Let $G=(V, E)$ be a simple graph with vertex set $V=$ $\left\{v_{1}, v_{2}, \ldots, v_{n}\right\}$ and edge set $E$. Let $d\left(v_{i}\right)$ be the degree of the vertex $v_{i} \in V(G)$ $(i=1,2, \ldots, n)$, and $D=D(G)=\operatorname{diag}\left(d\left(v_{1}\right), d\left(v_{2}\right), \ldots, d\left(v_{n}\right)\right)$ be the diagonal matrix of vertex degrees. The Laplacian matrix $L(G)=D(G)-A(G)$ is the difference between $D(G)$ and the adjacency matrix $A(G)$. It is easy to see that $L(G)$ is a positive semidefinite symmetric matrix with the smallest eigenvalue 0 and the corresponding eigenvector is the all ones column vector, which is denoted by $e$. Denote its eigenvalues by

$$
\mu_{1}(G) \geq \mu_{2}(G) \geq \cdots \geq \mu_{n}(G)=0
$$

which are always enumerated in non-increasing order and repeated according to their multiplicity. Fiedler [4] showed that the second smallest eigenvalue of $L(G)$ is 0 if and only if $G$ is disconnected. Thus, the second smallest eigenvalue of $L(G)$ is popularly known as the algebraic connectivity of $G$ and is usually denoted by $\alpha(G)$. Let $P_{n}$ and $C_{n}$ be the path and the cycle on $n$ vertices, respectively. It is a known fact that

$$
\alpha\left(P_{n}\right)=4 \sin ^{2} \frac{\pi}{2 n} \text { and } \alpha\left(C_{n}\right)=4 \sin ^{2} \frac{\pi}{n} .
$$

\footnotetext{
* Received by the editors on February 18, 2011. Accepted for publication on August 25, 2011. Handling Editor: Xingzhi Zhan.

${ }^{\dagger}$ Department of Mathematics, Hong Kong Baptist University, Kowloon Tong, Hong Kong, P.R. China (wcshiu@hkbu.edu.hk). Supported by FRG, Hong Kong Baptist University.

${ }^{\ddagger}$ Department of Applied Mathematics, China University of Petroleum, Dongying, Shandong, P.R. China (jimingguo@hotmail.com). Supported by the National Science Foundation of China (No. 10871204).

$\S$ Department of Mathematics and Information Science, Zhangzhou Normal University, Zhangzhou, Fujian, P.R. China (ptjxli@hotmail.com). Supported by the National Science Foundation of China (No. 11101358).
} 
Let $Y \in \mathbb{R}^{n}$ be a column vector. It will be convenient to assume that the entry $y_{i}$ of $Y$ is corresponding to the vertex $v_{i}$ of $G$. Such a $Y$ is sometimes called a valuation of the graph $G$ (see, [11]). In the following, $y_{i}$ will be written as $Y\left(v_{i}\right)$ for convenient. If $X$ is a unit eigenvector of $G$ corresponding to $\alpha(G)$, we commonly call it a Fiedler vector of $G$. It is obvious that $X^{T} e=0$ and

$$
\alpha(G)=X^{T} L(G) X=\sum_{v_{i} v_{j} \in E}\left(X\left(v_{i}\right)-X\left(v_{j}\right)\right)^{2}=\min _{\substack{Y \in \mathbb{R}^{n} \backslash\{0\} \\ Y^{T} e=0}} \frac{Y^{T} L(G) Y}{Y^{T} Y} .
$$

Furthermore, from $L(G) X=\alpha(G) X$, we also have the set of equations below, known as eigenvalue equations of $G$ :

$$
(d(v)-\alpha(G)) X(v)=\sum_{u \in N(v)} X(u) \text { for } v \in V(G),
$$

where $N_{G}(v)$ (or $N(v)$ for short) denotes the set of vertices which are adjacent to $v$ in $G$.

A caterpillar unicyclic graph is a unicyclic graph in which the removal of all pendant vertices makes it a cycle. Let $C_{g}=v_{1} v_{2} \cdots v_{g} v_{1}$ be a cycle with length $g$, where $v_{i} v_{i+1} \in E\left(C_{g}\right)$ for $i=1,2, \ldots, g-1$ and $v_{g} v_{1} \in E\left(C_{g}\right)$, and let $C_{g ; i_{1}, i_{2}, \ldots, i_{k}}^{n_{1}, n_{2}, \ldots, n_{k}}$ be the caterpillar unicyclic graph obtained from $C_{g}$ by attaching $n_{j}$ pendant edges at $v_{i_{j}}\left(1 \leq i_{1}<\cdots<i_{k} \leq g\right)$, respectively. By symmetry, we may always assume that $i_{1}=1$. For example, $C_{g ; 1,\left\lfloor\frac{g}{2}\right\rfloor+1}^{n_{1}, n_{2}}$ and $C_{g ; 1,\left\lfloor\frac{g}{2}\right\rfloor+1,\left\lfloor\frac{g}{2}\right\rfloor+2}^{n_{1}, n_{2}, n_{2}}$ (see Fig. 1.1) are two caterpillar unicyclic graphs which will be used in the next section. If $C_{g ; i_{1}, i_{2}, \ldots, i_{k}}^{n_{1}, n_{2}, \ldots, n_{k}}$ has $n$ vertices, then it is easy to see that $n_{1}+n_{2}+\cdots+n_{k}=n-g$.
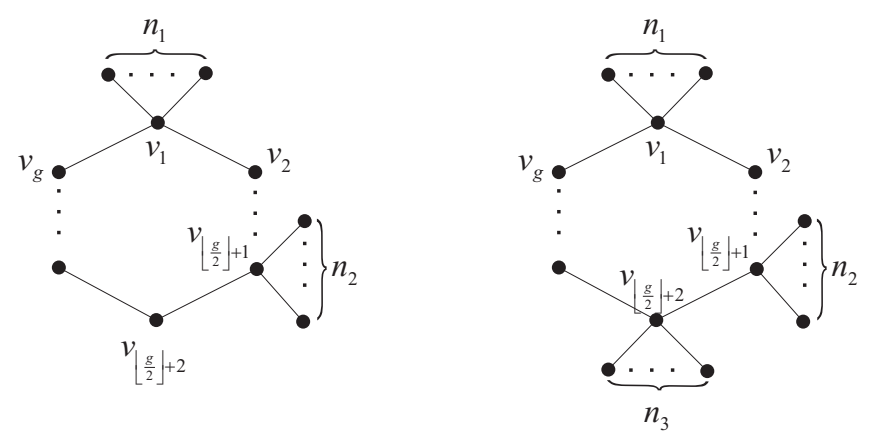

FIG. 1.1. Two caterpillar unicyclic graphs $C_{g ; 1,\left\lfloor\frac{g}{2}\right\rfloor+1}^{n_{1}, n_{2}}$ and $C_{g ; 1,\left\lfloor\frac{g}{2}\right\rfloor+1,\left\lfloor\frac{g}{2}\right\rfloor+2}^{n_{1}, n_{2}, n_{2}}$.

In [1], Fallat and Kirkland proved that for some choice of the parameters $n_{1}$, $n_{2}, \ldots, n_{g}$, the graph $C_{g ; 1,2, \ldots, g}^{n_{1}, n_{2}, \ldots, n_{g}}$ maximizes the algebraic connectivity over the 
class of unicyclic graphs with girth $g$. In particular, they proved that among all unicyclic graphs on $n$ vertices with girth 3 , the graph $C_{3 ; 1}^{n-3}$ has the maximum algebraic connectivity. In [2], Fallat, Kirkland and Pati proved that the graph $C_{4 ; 1}^{n-4}$ has the maximum algebraic connectivity among all unicyclic graphs on $n$ vertices with girth 4. Furthermore, they proved that there is an $N$ such that for each $n>N$, the graph $C_{g ; 1}^{n-g}$ has the maximum algebraic connectivity among all unicyclic graphs on $n$ vertices with girth $g$. On the other hand, when $g$ is large relative to $n$, they showed that this graph does not maximize the algebraic connectivity. For the minimum algebraic connectivity, Guo [7] proved that the graph $C_{n, g}$ has the minimum algebraic connectivity among all connected graphs with girth $g$, where $C_{n, g}$ is called the lollipop graph, which is obtained by appending a $g$-cycle $C_{g}$ to a pendant vertex of a path on $n-g$ vertices. This confirmes the conjecture proposed by Fallat and Kirkland (see [1], [3]).

In this paper, we prove that the graph $C_{g ; 1,\left\lfloor\frac{n}{2}\right\rfloor+1}^{\left\lceil\frac{n-g}{2}\right\rceil,\left\lfloor\frac{n-g}{2}\right\rfloor}$ has the minimum algebraic connectivity among all caterpillar unicyclic graphs on $n$ vertices with girth $g$.

Throughout this paper, we shall denote by $\Phi(B)=\Phi(B ; x)=\operatorname{det}(x I-B)$ the characteristic polynomial of the square matrix $B$. In particular, if $B=L(G)$, we write $\Phi(L(G))$ by $\Phi(G ; x)$ or simply by $\Phi(G)$ and call $\Phi(G)$ the Laplacian characteristic polynomial of $G$.

2. Lemmas and results. Let $G$ be a graph and let $G^{\prime}=G+e$ be the graph obtained from $G$ by inserting a new edge $e$ into $G$. The following lemma follows from Courant-Weyl inequalities (see [9]).

Lemma 2.1. The Laplacian eigenvalues of $G$ and $G^{\prime}$ interlace, that is,

$$
\mu_{1}\left(G^{\prime}\right) \geq \mu_{1}(G) \geq \mu_{2}\left(G^{\prime}\right) \geq \mu_{2}(G) \geq \cdots \geq \mu_{n}\left(G^{\prime}\right)=\mu_{n}(G)=0 .
$$

By Lemma 2.1, we immediately have the following:

Corollary 2.2. Let $G$ be a connected graph and $v$ be a pendant vertex of $G$. Then $\alpha(G) \leq \alpha(G-v)$.

The following inequalities are known as Cauchy's inequalities and the whole theorem is also known as interlacing theorem [9].

Lemma 2.3. Let $A$ be a Hermitian matrix with eigenvalues $\lambda_{1} \geq \lambda_{2} \geq \cdots \geq \lambda_{n}$ and $B$ be a principal sub-matrix of $A$. Let $B$ has eigenvalues $\rho_{1} \geq \rho_{2} \geq \cdots \geq \rho_{m}$ $(m \leq n)$. Then the inequalities $\lambda_{n-m+i} \leq \rho_{i} \leq \lambda_{i}$ hold for $i=1,2, \ldots, m$.

LEMMA 2.4. [5] Let $G_{1}=\left(V, E_{1}\right)$ be a graph on $n$ vertices and $G_{2}=\left(V, E_{2}\right)$ be 
a graph obtained from $G_{1}$ by removing an edge and adding a new edge that was not there before. Then

$$
\mu_{i}\left(G_{1}\right) \geq \mu_{i+1}\left(G_{2}\right) \text { and } \mu_{i}\left(G_{2}\right) \geq \mu_{i+1}\left(G_{1}\right) \text { for } 1 \leq i \leq n-1 .
$$

Lemma 2.5. [8] Suppose that $g \geq 4$. Then $\alpha\left(C_{n, g}\right)>\alpha\left(C_{n, g-1}\right)$.

For $U \subseteq V(G)$, let $L_{U}(G)$ be the principal sub-matrix of $L(G)$ formed by deleting the rows and columns corresponding to all vertices in $U$. If $U=\{v\}$, then we simple write $L_{U}(G)$ as $L_{v}(G)$. Let $H_{n}$ be the matrix of order $n$ obtained from $L\left(P_{n+2}\right)$ by deleting the rows and columns corresponding to two end vertices of $P_{n+2}$.

Lemma 2.6. [7] Set $\Phi\left(P_{0}\right)=0, \Phi\left(H_{0}\right)=1$. Then we have

(1) $\Phi\left(P_{n}\right)=x \Phi\left(H_{n-1}\right),(n \geq 1)$;

(2) $\Phi\left(P_{m}\right) \Phi\left(P_{n}\right)-\Phi\left(P_{m-1}\right) \Phi\left(P_{n+1}\right)=\Phi\left(P_{m-1}\right) \Phi\left(P_{n-1}\right)-\Phi\left(P_{m-2}\right) \Phi\left(P_{n}\right), \quad(m \geq$ $2, n \geq 1)$.

Corollary 2.7. For $m \geq k+1, n \geq 1$,

$\Phi\left(P_{m}\right) \Phi\left(P_{n}\right)-\Phi\left(P_{m-k}\right) \Phi\left(P_{n+k}\right)=\Phi\left(P_{m-1}\right) \Phi\left(P_{n-1}\right)-\Phi\left(P_{m-k-1}\right) \Phi\left(P_{n+k-1}\right)$.

Proof. From Lemma 2.6, we have

$$
\begin{aligned}
& \Phi\left(P_{m}\right) \Phi\left(P_{n}\right)-\Phi\left(P_{m-k}\right) \Phi\left(P_{n+k}\right) \\
= & \Phi\left(P_{m}\right) \Phi\left(P_{n}\right)-\Phi\left(P_{m-1}\right) \Phi\left(P_{n+1}\right)+\Phi\left(P_{m-1}\right) \Phi\left(P_{n+1}\right) \\
& -\Phi\left(P_{m-2}\right) \Phi\left(P_{n+2}\right)+\Phi\left(P_{m-2}\right) \Phi\left(P_{n+2}\right)-\cdots-\Phi\left(P_{m-k+1}\right) \Phi\left(P_{n+k-1}\right) \\
& +\Phi\left(P_{m-k+1}\right) \Phi\left(P_{n+k-1}\right)-\Phi\left(P_{m-k}\right) \Phi\left(P_{n+k}\right) \\
= & \Phi\left(P_{m-1}\right) \Phi\left(P_{n-1}\right)-\Phi\left(P_{m-2}\right) \Phi\left(P_{n}\right)+\Phi\left(P_{m-2}\right) \Phi\left(P_{n}\right)-\Phi\left(P_{m-3}\right) \Phi\left(P_{n+1}\right) \\
& +\Phi\left(P_{m-3}\right) \Phi\left(P_{n+1}\right)-\cdots-\Phi\left(P_{m-k}\right) \Phi\left(P_{n+k-2}\right) \\
& +\Phi\left(P_{m-k}\right) \Phi\left(P_{n+k-2}\right)-\Phi\left(P_{m-k-1}\right) \Phi\left(P_{n+k-1}\right) \\
= & \Phi\left(P_{m-1}\right) \Phi\left(P_{n-1}\right)-\Phi\left(P_{m-k-1}\right) \Phi\left(P_{n+k-1}\right) .
\end{aligned}
$$

Suppose $G_{1}$ and $G_{2}$ are two disjoint graphs. $u \in V\left(G_{1}\right)$ and $v \in V\left(G_{2}\right)$. Let $G=G_{1} u: v G_{2}$ be the graph obtained by joining the vertex $u$ of the graph $G_{1}$ to the vertex $v$ of the graph $G_{2}$ with an edge.

Lemma 2.8. [6] Let $G_{1}$ and $G_{2}$ be two disjoint graphs. Then

$$
\Phi\left(G_{1} u: v G_{2}\right)=\Phi\left(G_{1}\right) \Phi\left(G_{2}\right)-\Phi\left(G_{1}\right) \Phi\left(L_{v}\left(G_{2}\right)\right)-\Phi\left(L_{u}\left(G_{1}\right)\right) \Phi\left(G_{2}\right) .
$$


By a similar argument as the proof of Lemma 2.8, which was shown in [6], we also have the following result.

Corollary 2.9. Let $G_{1}$ and $G_{2}$ be two disjoint graphs. Suppose $w$ is a vertex of $G_{1}$ which is different from $u$. Then

$$
\begin{aligned}
\Phi\left(L_{w}\left(G_{1} u: v G_{2}\right)\right)= & \Phi\left(L_{w}\left(G_{1}\right)\right) \Phi\left(G_{2}\right)-\Phi\left(L_{w}\left(G_{1}\right)\right) \Phi\left(L_{v}\left(G_{2}\right)\right) \\
& -\Phi\left(L_{\{w, u\}}\left(G_{1}\right)\right) \Phi\left(G_{2}\right) .
\end{aligned}
$$

Let $G$ and $H$ be two disjoint graphs with $|V(G)|=s \geq 2,|V(H)|=t \geq 2$. Let $u \in V(G)$ and $r \in V(H)$. Let $G u \cdot r H$ be the graph obtained from $G$ and $H$ by identifying the two vertices $u$ and $r$ (suppose that the new vertex is still $u$ ). It is easy to see that $G u \cdot r H$ has $n=s+t-1$ vertices.

Lemma 2.10. Suppose $u$ and $v$ are two distinct vertices of $G$. Suppose $X$ is a Fiedler vector of $G u \cdot r H$. If $(X(v)-X(u)) \sum_{\substack{w \in V(H) \\ w \neq r}} X(w) \geq 0$, then $\alpha(G u \cdot r H) \geq$ $\alpha(G v \cdot r H)$. Moreover, the inequality is strict if $X(u) \neq X(v)$.

Proof. Let $Y$ be a valuation of $G v \cdot r H$ defined by

$$
Y(w)= \begin{cases}X(w)-\frac{(t-1)(X(v)-X(u))}{n}, & w \in V(G) \\ X(w)+\frac{s(X(v)-X(u))}{n}, & w \in V(H), w \neq r .\end{cases}
$$

It is easy to see that $Y^{T} e=0, Y^{T} L(G v \cdot r H) Y=X^{T} L(G u \cdot r H) X=\alpha(G u \cdot r H)$ and

$$
\begin{aligned}
Y^{T} Y= & X^{T} X-2 \sum_{w \in V(G)} \frac{(t-1)(X(v)-X(u))}{n} X(w)+\frac{s(t-1)^{2}(X(v)-X(u))^{2}}{n^{2}} \\
& +2 \sum_{\substack{w \in V(H) \\
w \neq r}} \frac{s(X(v)-X(u))}{n} X(w)+\frac{(t-1) s^{2}(X(v)-X(u))^{2}}{n^{2}} \\
= & 1+2(X(v)-X(u)) \sum_{\substack{w \in V(H) \\
w \neq r}} X(w)+\frac{s(t-1)(X(v)-X(u))^{2}}{n} \\
\geq & 1 .
\end{aligned}
$$

Clearly, the inequality is strict if $X(v) \neq X(u)$.

Thus, we have

$$
\alpha(G u \cdot r H)=X^{T} L(G u \cdot r H) X \geq \frac{Y^{T} L(G v \cdot r H) Y}{Y^{T} Y} \geq \alpha(G v \cdot r H),
$$


and the inequality is strict if $X(v) \neq X(u)$.

From Lemma 2.10, we immediately have the following.

COROLlary 2.11. Let $u, v$ be two vertices of a connected graph $G$ and there exist $s$ pendant edges $u u_{1}, u u_{2}, \ldots, u u_{s}$ at $u$. Suppose $X$ is a Fiedler vector of $G$. Let $G^{\prime}=G-u u_{1}-u u_{2}-\cdots-u u_{s}+v u_{1}+v u_{2}+\cdots+v u_{s}$. If $X(v) \geq X(u) \geq 0$, then $\alpha(G) \geq \alpha\left(G^{\prime}\right)$. Moreover, the inequality is strict if $X(v) \neq X(u)$.

LEMma 2.12. Let $C_{g ; 1,\left\lfloor\frac{g}{2}\right\rfloor+1}^{n_{1}, n_{2}}$ be the caterpillar unicyclic graph defined in Section 1. Then for $n \geq g+1$,

$$
\alpha\left(C_{g ; 1,\left\lfloor\frac{g}{2}\right\rfloor+1}^{n_{1}, n_{2}}\right)<\alpha\left(P_{g-\left\lfloor\frac{g}{2}\right\rfloor}\right) .
$$

Proof. From Corollary 2.2 and Lemma 2.5, we have

$$
\begin{aligned}
\alpha\left(C_{g ; 1,\left\lfloor\frac{g}{2}\right\rfloor+1}^{n_{1}, n_{2}}\right) \leq \alpha\left(C_{g+1, g}\right) & <\alpha\left(C_{g+1}\right)=4 \sin ^{2} \frac{\pi}{g+1} \\
& \leq 4 \sin ^{2} \frac{\pi}{2\left(g-\left\lfloor\frac{g}{2}\right\rfloor\right)}=\alpha\left(P_{g-\left\lfloor\frac{g}{2}\right\rfloor}\right)
\end{aligned}
$$

REMARK 1. Since $\alpha\left(P_{n}\right)$ is a decreasing function on $n, \alpha\left(C_{g ; 1,\left\lfloor\frac{g}{2}\right\rfloor+1}^{n_{1}, n_{2}}\right)<\alpha\left(P_{j}\right)$ for $j \leq g-\left\lfloor\frac{g}{2}\right\rfloor$.

LEMma 2.13. [10] Let $G$ be a connected graph with a cut vertex $v$. Then $\alpha(G) \leq 1$, the equality holds if and only if $v$ is adjacent to every vertex of $G$.

LEMMA 2.14. Let $C_{g ; 1,\left\lfloor\frac{g}{2}\right\rfloor+1}^{n_{1}, n_{2}}$ and $C_{g ; 1, i}^{n_{1}, n_{2}}\left(2 \leq i \leq\left\lfloor\frac{g}{2}\right\rfloor\right)$ be the caterpillar unicyclic graphs defined in Section 1. Then for $n_{1}, n_{2} \geq 1$,

$$
\alpha\left(C_{g ; 1,\left\lfloor\frac{g}{2}\right\rfloor+1}^{n_{1}, n_{2}}\right) \leq \mu_{n-2}\left(C_{g ; 1, i}^{n_{1}, n_{2}}\right) .
$$

Proof. If $\mu_{n-2}\left(C_{g ; 1, i}^{n_{1}, n_{2}}\right) \geq 1$, then the result follows from Lemma 2.13. Thus, in the following, we assume that $\mu_{n-2}\left(C_{g ; 1, i}^{n_{1}, n_{2}}\right)<1$. From Corollary 2.2 and Lemma 2.3, we have

$$
\alpha\left(C_{g ; 1,\left\lfloor\frac{g}{2}\right\rfloor+1}^{n_{1}, n_{2}}\right) \leq \alpha\left(C_{g ; 1,\left\lfloor\frac{g}{2}\right\rfloor+1}^{n_{1}, 0}\right)=\alpha\left(C_{g ; 1}^{n_{1}}\right) \leq \lambda_{g+n_{1}-2}\left(L_{v_{i}}\left(C_{g ; 1}^{n_{1}}\right)\right) \leq \mu_{n-2}\left(C_{g ; 1, i}^{n_{1}, n_{2}}\right),
$$

where $\lambda_{g+n_{1}-2}\left(L_{v_{i}}\left(C_{g ; 1}^{n_{1}}\right)\right)$ denotes the second smallest eigenvalue of $L_{v_{i}}\left(C_{g ; 1}^{n_{1}}\right)$.

LEMMA 2.15. For $2 \leq i \leq\left\lfloor\frac{g}{2}\right\rfloor$ and $n_{1}, n_{2} \geq 1$, we have

$$
\alpha\left(C_{g ; 1, i}^{n_{1}, n_{2}}\right)>\alpha\left(C_{g ; 1,\left\lfloor\frac{g}{2}\right\rfloor+1}^{n_{1}, n_{2}}\right) .
$$


Proof. Applying Lemma 2.8 and Corollary 2.9 for $n_{1}$ times, we have

$$
\begin{aligned}
& \Phi\left(C_{g ; 1, i}^{n_{1}, n_{2}}\right)-\Phi\left(C_{g ; 1,\left\lfloor\frac{g}{2}\right\rfloor+1}^{n_{1}, n_{2}}\right) \\
= & (x-1) \Phi\left(C_{g ; 1, i}^{n_{1}-1, n_{2}}\right)-x(x-1)^{n_{1}-1} \Phi\left(L_{v_{1}}\left(C_{g ; 1, i}^{0, n_{2}}\right)\right)-(x-1) \Phi\left(C_{g ; 1,\left\lfloor\frac{g}{2}\right\rfloor+1}^{n_{1}-1, n_{2}}\right) \\
& +x(x-1)^{n_{1}-1} \Phi\left(L_{v_{1}}\left(C_{g ; 1,\left\lfloor\frac{g}{2}\right\rfloor+1}^{0, n_{2}}\right)\right) \\
& \vdots \quad \vdots \\
= & (x-1)^{n_{1}} \Phi\left(C_{g ; 1, i}^{0, n_{2}}\right)-n_{1} x(x-1)^{n_{1}-1} \Phi\left(L_{v_{1}}\left(C_{g ; 1, i}^{0, n_{2}}\right)\right)-(x-1)^{n_{1}} \Phi\left(C_{g ; 1,\left\lfloor\frac{g}{2}\right\rfloor+1}^{0, n_{2}}\right) \\
& +n_{1} x(x-1)^{n_{1}-1} \Phi\left(L_{v_{1}}\left(C_{g ; 1,\left\lfloor\frac{g}{2}\right\rfloor+1}^{0, n_{2}}\right)\right) .
\end{aligned}
$$

Note that $C_{g ; 1, i}^{0, n_{2}}=C_{g ; 1,\left\lfloor\frac{g}{2}\right\rfloor+1}^{0, n_{2}}=C_{g ; 1}^{n_{2}}$. Then from the above equation, we have

$$
\Phi\left(C_{g ; 1, i}^{n_{1}, n_{2}}\right)-\Phi\left(C_{g ; 1,\left\lfloor\frac{g}{2}\right\rfloor+1}^{n_{1}, n_{2}}\right)=n_{1} x(x-1)^{n_{1}-1}\left[\Phi\left(L_{v_{1}}\left(C_{g ; 1,\left\lfloor\frac{g}{2}\right\rfloor+1}^{0, n_{2}}\right)\right)-\Phi\left(L_{v_{1}}\left(C_{g ; 1, i}^{0, n_{2}}\right)\right)\right]
$$

Applying Corollary 2.9 again, we have

$$
\begin{aligned}
& \Phi\left(L_{v_{1}}\left(C_{g ; 1,\left\lfloor\frac{g}{2}\right\rfloor+1}^{0, n_{2}}\right)\right)-\Phi\left(L_{v_{1}}\left(C_{g ; 1, i}^{0, n_{2}}\right)\right) \\
= & (x-1) \Phi\left(L_{v_{1}}\left(C_{g ; 1,\left\lfloor\frac{g}{2}\right\rfloor+1}^{0, n_{2}-1}\right)\right)-x(x-1)^{n_{2}-1} \Phi\left(H_{\left\lfloor\frac{g}{2}\right\rfloor-1}\right) \Phi\left(H_{g-\left\lfloor\frac{g}{2}\right\rfloor-1}\right) \\
& -(x-1) \Phi\left(L_{v_{1}}\left(C_{g ; 1, i}^{0, n_{2}-1}\right)\right)+x(x-1)^{n_{2}-1} \Phi\left(H_{i-2}\right) \Phi\left(H_{g-i}\right) \\
& \vdots \\
= & (x-1)^{n_{2}} \Phi\left(L_{v_{1}}\left(C_{g ; 1,\left\lfloor\frac{g}{2}\right\rfloor+1}^{0,0}\right)\right)-n_{2} x(x-1)^{n_{2}-1} \Phi\left(H_{\left\lfloor\frac{g}{2}\right\rfloor-1}\right) \Phi\left(H_{g-\left\lfloor\frac{g}{2}\right\rfloor-1}\right) \\
& -(x-1)^{n_{2}} \Phi\left(L_{v_{1}}\left(C_{g ; 1, i}^{0,0}\right)\right)+n_{2} x(x-1)^{n_{2}-1} \Phi\left(H_{i-2}\right) \Phi\left(H_{g-i}\right)
\end{aligned}
$$

Note that $L_{v_{1}}\left(C_{g ; 1,\left\lfloor\frac{g}{2}\right\rfloor+1}^{0,0}\right)=L_{v_{1}}\left(C_{g ; 1, i}^{0,0}\right)=L_{v_{1}}\left(C_{g}\right)=\Phi\left(H_{g-1}\right)$. Thus, from the above equation, we have

$$
\begin{aligned}
& \Phi\left(L_{v_{1}}\left(C_{g ; 1,\left\lfloor\frac{g}{2}\right\rfloor+1}^{0, n_{2}}\right)\right)-\Phi\left(L_{v_{1}}\left(C_{g ; 1, i}^{0, n_{2}}\right)\right) \\
= & n_{2} x(x-1)^{n_{2}-1}\left[\Phi\left(H_{i-2}\right) \Phi\left(H_{g-i}\right)-\Phi\left(H_{\left\lfloor\frac{g}{2}\right\rfloor-1}\right) \Phi\left(H_{g-\left\lfloor\frac{g}{2}\right\rfloor-1}\right)\right] .
\end{aligned}
$$

Substituting (2.2) into (2.1), and from Lemma 2.6 and Corollary 2.7, we have

$$
\begin{aligned}
& \Phi\left(C_{g ; 1, i}^{n_{1}, n_{2}}\right)-\Phi\left(C_{g ; 1,\left\lfloor\frac{g}{2}\right\rfloor+1}^{n_{1}, n_{2}}\right) \\
= & n_{1} n_{2} x^{2}(x-1)^{n_{1}+n_{2}-2}\left[\Phi\left(H_{i-2}\right) \Phi\left(H_{g-i}\right)-\Phi\left(H_{\left\lfloor\frac{g}{2}\right\rfloor-1}\right) \Phi\left(H_{g-\left\lfloor\frac{g}{2}\right\rfloor-1}\right)\right] \\
= & n_{1} n_{2}(x-1)^{n_{1}+n_{2}-2}\left[\Phi\left(P_{i-1}\right) \Phi\left(P_{g-i+1}\right)-\Phi\left(P_{\left\lfloor\frac{g}{2}\right\rfloor}\right) \Phi\left(P_{g-\left\lfloor\frac{g}{2}\right\rfloor}\right)\right] \\
= & -n_{1} n_{2}(x-1)^{n_{1}+n_{2}-2} \Phi\left(P_{\left\lfloor\frac{g}{2}\right\rfloor-i+1}\right) \Phi\left(P_{g-\left\lfloor\frac{g}{2}\right\rfloor-i+1}\right) .
\end{aligned}
$$


Let $\alpha=\alpha\left(C_{g ; 1,\left\lfloor\frac{g}{2}\right\rfloor+1}^{n_{1}, n_{2}}\right)$. From Lemma 2.12, we have

$$
\begin{aligned}
& (-1)^{n_{1}+n_{2}+g-1}\left[\Phi\left(C_{g ; 1, i}^{n_{1}, n_{2}}, \alpha\right)-\Phi\left(C_{g ; 1,\left\lfloor\frac{g}{2}\right\rfloor+1}^{n_{1}, n_{2}}, \alpha\right)\right] \\
= & (-1)^{n_{1}+n_{2}+g}\left[n_{1} n_{2}(\alpha-1)^{n_{1}+n_{2}-2} \Phi\left(P_{\left\lfloor\frac{g}{2}\right\rfloor-i+1}, \alpha\right) \Phi\left(P_{g-\left\lfloor\frac{g}{2}\right\rfloor-i+1}, \alpha\right)\right] .
\end{aligned}
$$

By Remark 1 and the fact $0<\alpha<1$, the above expression is positive. Note that $n_{1}+n_{2}+g=n$ is the order of the graph $C_{g ; 1, i}^{n_{1}, n_{2}}$. So $(-1)^{n-1} \Phi\left(C_{g ; 1, i}^{n_{1}, n_{2}}, \alpha\right)>0$. Thus, from Lemma 2.14, we have $\alpha\left(C_{g ; 1, i}^{n_{1}, n_{2}}\right)>\alpha\left(C_{g ; 1,\left\lfloor\frac{g}{2}\right\rfloor+1}^{n_{1}, n_{2}}\right)$.

LEMma 2.16. For $n_{1} \geq n_{2}+2, \alpha\left(C_{g ; 1,\left\lfloor\frac{g}{2}\right\rfloor+1}^{n_{1}, n_{2}}\right)>\alpha\left(C_{g ; 1,\left\lfloor\frac{g}{2}\right\rfloor+1}^{n_{1}-1, n_{2}+1}\right)$.

Proof. We separate the proof into two cases.

Case 1. $n_{2} \geq 1$.

Applying Lemma 2.8 and Corollary 2.9 for several times, we have

$$
\begin{aligned}
& \Phi\left(C_{g ; 1,\left\lfloor\frac{g}{2}\right\rfloor+1}^{n_{1}-1, n_{2}+1}\right)-\Phi\left(C_{g ; 1,\left\lfloor\frac{g}{2}\right\rfloor+1}^{n_{1}, n_{2}}\right) \\
= & (x-1) \Phi\left(C_{g ; 1,\left\lfloor\frac{g}{2}\right\rfloor+1}^{n_{1}-1, n_{2}}\right)-x(x-1)^{n_{2}} \Phi\left(L_{v_{\left\lfloor\frac{g}{2}\right\rfloor+1}}\left(C_{g ; 1,\left\lfloor\frac{g}{2}\right\rfloor+1}^{n_{1}-1,0}\right)\right) \\
& -(x-1) \Phi\left(C_{g ; 1,\left\lfloor\frac{g}{2}\right\rfloor+1}^{n_{1}-1, n_{2}}\right)+x(x-1)^{n_{1}-1} \Phi\left(L_{v_{1}}\left(C_{g ; 1,\left\lfloor\frac{g}{2}\right\rfloor+1}^{0, n_{2}}\right)\right) \\
= & x(x-1)^{n_{1}-1} \Phi\left(L_{v_{1}}\left(C_{g ; 1,\left\lfloor\frac{g}{2}\right\rfloor+1}^{0, n_{2}}\right)\right)-x(x-1)^{n_{2}} \Phi\left(L_{v_{\left\lfloor\frac{g}{2}\right\rfloor+1}}\left(C_{g ; 1,\left\lfloor\frac{g}{2}\right\rfloor+1}^{n_{1}-1,0}\right)\right) \\
= & x(x-1)^{n_{1}} \Phi\left(L_{v_{1}}\left(C_{g ; 1,\left\lfloor\frac{g}{2}\right\rfloor+1}^{0, n_{2}-1}\right)\right)-x^{2}(x-1)^{n_{1}+n_{2}-2} \Phi\left(H_{\left\lfloor\frac{g}{2}\right\rfloor-1}\right) \Phi\left(H_{g-\left\lfloor\frac{g}{2}\right\rfloor-1}\right) \\
& -x(x-1)^{n_{2}+1} \Phi\left(L_{v_{\left\lfloor\frac{g}{2}\right\rfloor+1}}\left(C_{g ; 1,\left\lfloor\frac{g}{2}\right\rfloor+1}^{n_{1}-2,0}\right)\right) \\
& +x^{2}(x-1)^{n_{1}+n_{2}-2} \Phi\left(H_{\left\lfloor\frac{g}{2}\right\rfloor-1}\right) \Phi\left(H_{g-\left\lfloor\frac{g}{2}\right\rfloor-1}\right) \\
= & x(x-1)^{n_{1}} \Phi\left(L_{v_{1}}\left(C_{g ; 1,\left\lfloor\frac{g}{2}\right\rfloor+1}^{0, n_{2}-1}\right)\right)-x(x-1)^{n_{2}+1} \Phi\left(L_{v_{\left\lfloor\frac{g}{2}\right\rfloor+1}}\left(C_{g ; 1,\left\lfloor\frac{g}{2}\right\rfloor+1}^{n_{1}-2,0}\right)\right) \\
= & x(x-1)^{n_{1}+n_{2}-1} \Phi\left(L_{v_{1}}\left(C_{g ; 1,\left\lfloor\frac{g}{2}\right\rfloor+1}^{0,0}\right)\right) \\
& -n_{2} x^{2}(x-1)^{n_{1}+n_{2}-2} \Phi\left(H_{\left\lfloor\frac{g}{2}\right\rfloor-1}\right) \Phi\left(H_{g-\left\lfloor\frac{g}{2}\right\rfloor-1}\right) \\
& -x(x-1)^{n_{1}+n_{2}-1} \Phi\left(L_{v_{\left\lfloor\frac{g}{2}\right\rfloor+1}}\left(C_{g ; 1,\left\lfloor\frac{g}{2}\right\rfloor+1}^{0,0}\right)\right) \\
& +\left(n_{1}-1\right) x^{2}(x-1)^{n_{1}+n_{2}-2} \Phi\left(H_{\left\lfloor\frac{g}{2}\right\rfloor-1}\right) \Phi\left(H_{g-\left\lfloor\frac{g}{2}\right\rfloor-1}\right) .
\end{aligned}
$$

Note that $L_{v_{1}}\left(C_{g ; 1,\left\lfloor\frac{g}{2}\right\rfloor+1}^{0,0}\right)=L_{v_{\left\lfloor\frac{g}{2}\right\rfloor+1}}\left(C_{g ; 1,\left\lfloor\frac{g}{2}\right\rfloor+1}^{0,0}\right)=L_{v_{1}}\left(C_{g}\right)$. Then from Lemma 2.6 and the above equation we have

$$
\begin{aligned}
& \Phi\left(C_{g ; 1,\left\lfloor\frac{g}{2}\right\rfloor+1}^{n_{1}-1, n_{2}+1}\right)-\Phi\left(C_{g ; 1,\left\lfloor\frac{g}{2}\right\rfloor+1}^{n_{1}, n_{2}}\right) \\
= & x^{2}(x-1)^{n_{1}+n_{2}-2}\left(n_{1}-n_{2}-1\right) \Phi\left(H_{\left\lfloor\frac{g}{2}\right\rfloor-1}\right) \Phi\left(H_{g-\left\lfloor\frac{g}{2}\right\rfloor-1}\right) \\
= & (x-1)^{n_{1}+n_{2}-2}\left(n_{1}-n_{2}-1\right) \Phi\left(P_{\left\lfloor\frac{g}{2}\right\rfloor}\right) \Phi\left(P_{g-\left\lfloor\frac{g}{2}\right\rfloor}\right) .
\end{aligned}
$$

Let $\alpha=\alpha\left(C_{g ; 1,\left\lfloor\frac{g}{2}\right\rfloor+1}^{n_{1}, n_{2}}\right)$. From Lemma 2.4, we have $\alpha \leq \mu_{n-2}\left(C_{g ; 1,\left\lfloor\frac{g}{2}\right\rfloor+1}^{n_{1}-1, n_{2}+1}\right)$. 
Thus, similar to the proof of Lemma 2.15, we have

$$
\begin{aligned}
& (-1)^{n_{1}+n_{2}+g-1}\left[\Phi\left(C_{g ; 1,\left\lfloor\frac{g}{2}\right\rfloor+1}^{n_{1}-1, n_{2}+1}, \alpha\right)-\Phi\left(C_{g ; 1,\left\lfloor\frac{g}{2}\right\rfloor+1}^{n_{1}, n_{2}}, \alpha\right)\right] \\
= & (-1)^{n_{1}+n_{2}+g-1}(\alpha-1)^{n_{1}+n_{2}-2}\left(n_{1}-n_{2}-1\right) \Phi\left(P_{\left\lfloor\frac{g}{2}\right\rfloor}, \alpha\right) \Phi\left(P_{g-\left\lfloor\frac{g}{2}\right\rfloor}, \alpha\right)<0 .
\end{aligned}
$$

Then, we have $\alpha\left(C_{g ; 1,\left\lfloor\frac{g}{2}\right\rfloor+1}^{n_{1}, n_{2}}\right)>\alpha\left(C_{g ; 1,\left\lfloor\frac{g}{2}\right\rfloor+1}^{n_{1}-1, n_{2}+1}\right)$.

Case 2. $n_{2}=0$.

From Lemma 2.8 and Corollary 2.9, we have

$$
\begin{aligned}
& \Phi\left(C_{g ; 1,\left\lfloor\frac{g}{2}\right\rfloor+1}^{n_{1}-1,1}\right)-\Phi\left(C_{g ; 1}^{n_{1}}\right) \\
= & (x-1) \Phi\left(C_{g ; 1,\left\lfloor\frac{g}{2}\right\rfloor+1}^{n_{1}-1,0}\right)-x \Phi\left(L_{v_{\left\lfloor\frac{g}{2}\right\rfloor+1}}\left(C_{g ; 1,\left\lfloor\frac{g}{2}\right\rfloor+1}^{n_{1}-1,0}\right)\right) \\
& -(x-1) \Phi\left(C_{g ; 1}^{n_{1}-1}\right)+x(x-1)^{n_{1}-1} \Phi\left(L_{v_{1}}\left(C_{g ; 1}^{0}\right)\right) \\
= & x(x-1)^{n_{1}-1} \Phi\left(L_{v_{1}}\left(C_{g ; 1}^{0}\right)\right)-x \Phi\left(L_{v_{\left\lfloor\frac{g}{2}\right\rfloor+1}}\left(C_{g ; 1,\left\lfloor\frac{g}{2}\right\rfloor+1}^{n_{1}-1,0}\right)\right) \\
= & x(x-1)^{n_{1}-1} \Phi\left(L_{v_{1}}\left(C_{g ; 1}^{0}\right)\right)-x(x-1)^{n_{1}-1} \Phi\left(L_{v_{\left\lfloor\frac{g}{2}\right\rfloor+1}}\left(C_{g ; 1,\left\lfloor\frac{g}{2}\right\rfloor+1}^{0,0}\right)\right) \\
& +\left(n_{1}-1\right) x^{2}(x-1)^{n_{1}-2} \Phi\left(H_{\left\lfloor\frac{g}{2}\right\rfloor-1}\right) \Phi\left(H_{g-\left\lfloor\frac{g}{2}\right\rfloor-1}\right) \\
= & \left(n_{1}-1\right) x^{2}(x-1)^{n_{1}-2} \Phi\left(H_{\left\lfloor\frac{g}{2}\right\rfloor-1}\right) \Phi\left(H_{g-\left\lfloor\frac{g}{2}\right\rfloor-1}\right) .
\end{aligned}
$$

By a similar argument as that of Case 1, the result follows.

Now we give the main result of this paper.

THEOREM 2.17. Let $G$ be a caterpillar unicyclic graph on $n$ vertices with girth g. Then

$$
\alpha(G) \geq \alpha\left(C_{g ; 1,\left\lfloor\frac{g}{2}\right\rfloor+1}^{\left\lceil\frac{n-g}{2}\right\rceil,\left\lfloor\frac{n-g}{2}\right\rfloor}\right)
$$

and the equality holds if and only if $G=C_{g ; 1,\left\lfloor\frac{g}{2}\right\rfloor+1}^{\left\lceil\frac{n-g}{2}\right\rceil,\left\lfloor\frac{n-g}{2}\right\rfloor}$.

Proof. Since $G$ is a caterpillar unicyclic graph on $n$ vertices with girth $g$, we may assume that

$$
G=C_{g ; i_{1}, i_{2}, \ldots, i_{k}}^{n_{1}, n_{2}, \ldots, n_{k}}, \quad n_{j} \geq 1 \text { for } 1 \leq j \leq k \leq g ; 1 \leq i_{1}<i_{2}<\cdots<i_{k} \leq g .
$$

For $k=1$, the result follows from Case 2 of the proof of Lemma 2.16. For $k=2$, the result follows from Lemmas 2.15 and 2.16 .

For $k=3$, let $X$ be a Fiedler vector of $G=C_{g ; i_{1}, i_{2}, i_{3}}^{n_{1}, n_{2}, n_{3}}$. Since $-X$ is also a Fiedler vector of $G$, without loss of generality, we may assume that there are at least two of $X\left(v_{i_{1}}\right), X\left(v_{i_{2}}\right)$ and $X\left(v_{i_{3}}\right)$ being nonnegative. By renumbering, we may assume that $G \cong C_{g ; 1, i, j}^{n_{1}, n_{2}, n_{3}}$ with $X\left(v_{j}\right) \geq X\left(v_{i}\right) \geq X\left(v_{1}\right)$ and $X\left(v_{i}\right) \geq 0$. Suppose $X\left(v_{j}\right)>X\left(v_{i}\right)$. Then by Corollary 2.11, $\alpha(G)>\alpha\left(C_{g ; 1, j}^{n_{1}, n_{2}+n_{3}}\right)$. By renumbering of the vertices, we 
may assume that $j \leq\left\lfloor\frac{g}{2}\right\rfloor+1$. By Lemmas 2.15 and 2.16 if necessary, we obtain the result.

So now we assume that $X\left(v_{j}\right)=X\left(v_{i}\right)$. If the distance between $v_{1}$ and $v_{i}$ or the distance between $v_{1}$ and $v_{j}$ less than $\left\lfloor\frac{g}{2}\right\rfloor$, then (by renumbering the vertices if necessary) we may assume that $i \leq\left\lfloor\frac{g}{2}\right\rfloor$. Then by Corollary 2.11, Lemmas 2.15 and 2.16 , we obtain that $\alpha(G) \geq \alpha\left(C_{g ; 1, i}^{n_{1}, n_{2}+n_{3}}\right)>\alpha\left(C_{g ; 1,\left\lfloor\frac{g}{2}\right\rfloor+1}^{n_{1}, n_{2}+n_{3}}\right) \geq \alpha\left(C_{g ; 1,\left\lfloor\frac{g}{2}\right\rfloor+1}^{\left\lfloor\frac{n-g}{2}\right\rfloor,\left\lceil\frac{n-g}{2}\right\rceil}\right)$.

Thus, we have to deal with the case that the distance between $v_{1}$ to both $v_{i}$ and $v_{j}$ are $\left\lfloor\frac{g}{2}\right\rfloor$. Note that the necessary condition for the occurrence of this case is $g$ being odd. So now $G \cong C_{g ; 1,\left\lfloor\frac{g}{2}\right\rfloor+1,\left\lfloor\frac{g}{2}\right\rfloor+2}^{n_{1}, n_{2}, n_{3}}$ with $X\left(v_{\left\lfloor\frac{g}{2}\right\rfloor+1}\right)=X\left(v_{\left\lfloor\frac{g}{2}\right\rfloor+2}\right)$. Note that

$$
\alpha(G)=X^{T} L\left(C_{g ; 1,\left\lfloor\frac{g}{2}\right\rfloor+1,\left\lfloor\frac{g}{2}\right\rfloor+2}^{n_{1}, n_{2}, n_{3}}\right) X=X^{T} L\left(C_{g ; 1,\left\lfloor\frac{g}{2}\right\rfloor+2}^{n_{1}, n_{2}+n_{3}}\right) X \geq \alpha\left(C_{g ; 1,\left\lfloor\frac{g}{2}\right\rfloor+2}^{n_{1}, n_{2}+n_{3}}\right) .
$$

Suppose that $\alpha(G)=\alpha\left(C_{g ; 1,\left\lfloor\frac{g}{2}\right\rfloor+2}^{n_{1}, n_{2}+n_{3}}\right)$. From the above equation we can see that $X$ is also a Fiedler vector of $C_{g ; 1,\left\lfloor\frac{g}{2}\right\rfloor+2}^{n_{1}, n_{2}+n_{3}}$. From the eigenvalue equations of $G$, we have

$$
\begin{aligned}
& \left(d_{G}\left(v_{\left\lfloor\frac{g}{2}\right\rfloor+1}\right)-\alpha(G)\right) X\left(v_{\left\lfloor\frac{g}{2}\right\rfloor+1}\right) \\
& =\sum_{w \in N\left(v_{\left\lfloor\frac{g}{2}\right\rfloor+1}\right)} X(w)=X\left(v_{\left\lfloor\frac{g}{2}\right\rfloor+1}\right)+X\left(v_{\left\lfloor\frac{g}{2}\right\rfloor}\right)+\sum_{\substack{w \in N\left(v\left\lfloor\frac{g}{w}\right\rfloor+1 \\
w \neq v\right.}} X(w) .
\end{aligned}
$$

Note that for $w \in N\left(v_{\left\lfloor\frac{g}{2}\right\rfloor+1}\right) \backslash\left\{v_{\left\lfloor\frac{g}{2}\right\rfloor}, v_{\left\lfloor\frac{g}{2}\right\rfloor+2}\right\},(1-\alpha(G)) X(w)=X\left(v_{\left\lfloor\frac{g}{2}\right\rfloor+1}\right)$. Thus, the above equation becomes

$$
X\left(v_{\left\lfloor\frac{g}{2}\right\rfloor}\right)=\left(d_{G}\left(v_{\left\lfloor\frac{g}{2}\right\rfloor+1}\right)-1-\alpha(G)-\frac{d_{G}\left(v_{\left\lfloor\frac{g}{2}\right\rfloor+1}\right)-2}{1-\alpha(G)}\right) X\left(v_{\left\lfloor\frac{g}{2}\right\rfloor+1}\right) .
$$

Similarly, from the eigenvalue equations of $C_{g ; 1,\left\lfloor\frac{g}{2}\right\rfloor+2}^{n_{1}, n_{2}+n_{3}}$, we have

$$
\left(2-\alpha\left(C_{g ; 1,\left\lfloor\frac{g}{2}\right\rfloor+2}^{n_{1}, n_{2}+n_{3}}\right)\right) X\left(v_{\left\lfloor\frac{g}{2}\right\rfloor+1}\right)=X\left(v_{\left\lfloor\frac{g}{2}\right\rfloor+2}\right)+X\left(v_{\left\lfloor\frac{g}{2}\right\rfloor}\right) .
$$

Then

$$
X\left(v_{\left\lfloor\frac{g}{2}\right\rfloor}\right)=\left(1-\alpha\left(C_{g ; 1,\left\lfloor\frac{g}{2}\right\rfloor+2}^{n_{1}, n_{2}+n_{3}}\right)\right) X\left(v_{\left\lfloor\frac{g}{2}\right\rfloor+1}\right) .
$$

Combining (2.3) and (2.4), we have

$$
\left(d_{G}\left(v_{\left\lfloor\frac{g}{2}\right\rfloor+1}\right)-2-\frac{d_{G}\left(v_{\left\lfloor\frac{g}{2}\right\rfloor+1}\right)-2}{1-\alpha(G)}\right) X\left(v_{\left\lfloor\frac{g}{2}\right\rfloor+1}\right)=0 .
$$

Since $n_{2} \geq 1, d_{G}\left(v_{\left\lfloor\frac{g}{2}\right\rfloor+1}\right)>2$. Thus, we have $X\left(v_{\left\lfloor\frac{g}{2}\right\rfloor+1}\right)=0$. From the eigenvalue equations of $G$, it is easy to see that $X=\mathbf{0}$. It yields a contradiction. So $\alpha(G)>$ $\alpha\left(C_{g ; 1,\left\lfloor\frac{g}{2}\right\rfloor+2}^{n_{1}, n_{2}+n_{3}}\right)$. 
For $k \geq 4$, from Corollary 2.11, there exists some caterpillar unicyclic graph, say $C_{g ; 1, i, j}^{n_{1}, n_{2}, n_{3}}$ for $n_{1}, n_{2}, n_{3} \geq 1$ and $1<i<j$, such that $\alpha(G) \geq \alpha\left(C_{g ; 1, i, j}^{n_{1}, n_{2}, n_{3}}\right)$. This case is referred to the case when $k=3$.

Hence, the proof is completed.

\section{REFERENCES}

[1] S. Fallat and S. Kirkland. Extremizing algebraic connectivity subject to graph theoretic constraints. Electron. J. Linear Algebra., 3:48-74, 1998.

[2] S. Fallat, S. Kirkland, and S. Pati. Maximizing algebraic connectivity over unicyclic graphs. Linear Multilinear Algebra, 51:221-241, 2003.

[3] S. Fallat, S. Kirkland, and S. Pati. Minimizing algebraic connectivity over connected graphs with fixed girth. Discrete Math., 254:115-142, 2002.

[4] M. Fiedler. Algebraic connectivity of graphs. Czech. Math. J., 23:298-305, 1973.

[5] R. Grone, R. Merris, and V.S. Sunder. The Laplacian spectrum of a graph. SIAM J. Matrix Anal. Appl., 11:218-238, 1990.

[6] J-M. Guo. On the second largest Laplacian eigenvalue of trees. Linear Algebra Appl., 404:251261, 2005.

[7] J-M. Guo. A conjecture on the algebraic connectivity of connected graphs with fixed girth. Discrete Math., 308:702-711, 2008.

[8] J-M. Guo, W.C. Shiu, and J. Li. The algebraic connectivity of lollipop graph. Linear Algebra Appl., 434:2204-2210, 2011.

[9] R.A. Horn and C.R. Johnson, Matrix Analysis, Cambridge University Press, New York, 1985.

[10] S. Kirkland, A bound on the algebraic connectivity of a graph in terms of the number of cutpoints, Linear Multilinear Algebra, 47:93-103, 2000.

[11] R. Merris, Laplacian graph eigenvectors, Linear Algebra Appl., 278:221-236, 1998. 